EPJ Web of Conferences 19, 04005 (2012)

DOI: $10.1051 /$ epjconf/20121904005

(C) Owned by the authors, published by EDP Sciences, 2012

\title{
Thick disk and pseudobulge formation in a clump cluster
}

\author{
S. Inoue $1,2, \mathrm{a}$ \\ ${ }^{1}$ Astronomical Institute, Tohoku University, Sendai 980-8578, Japan \\ ${ }^{2}$ Mullard Space Science Laboratory, University College London, Holmbury St. Mary, Dorking, \\ Surrey, RH5 6NT, UK
}

\begin{abstract}
Bulges in spiral galaxies have been supposed to be classified into two types: classical bulges or pseudobulges. Classical bulges are thought to form by galactic merger with bursty star formation, whereas pseudobulges are suggested to form by secular evolution. Noguchi $(1998,199)$ suggested another bulge formation scenario, 'clump-origin bulge' $[1,2]$. He demonstrated using a numerical simulation that a galactic disc suffers dynamical instability to form clumpy structures in the early stage of disc formation, then the clumps are sucked into the galactic centre by dynamical friction and merge into a single bulge at the centre. Therefore, clump-origin bulges may have their own unique properties. I perform a high-resolution $\mathrm{N}$-body/SPH simulation for the formation of the clump-origin bulge in an isolated galaxy model and study the formation of the clump-origin bulge. I find that the clump-origin bulge resembles pseudobulges in dynamical properties, a nearly exponential surface density profile, a barred boxy shape and a significant rotation. I also find that this bulge consists of old and metal-rich stars. These natures, old metal-rich population but pseudobulge-like structures, mean that the clump-origin bulge can not be simply classified into classical bulges nor pseudobulges. From these results, I discuss similarities of the clump-origin bulge to the Milky Way (MW) bulge.
\end{abstract}

\section{INTRODUCTION}

[3] has suggested that bulges in spiral galaxies can be classified into classical bulges or pseudobulges. Classical bulges are thought to form through galactic merger. Pseudobulges are discussed to form through secular evolution caused by non-axisymmetric structures in a galactic disc. [1,2] demonstrated that clumpy structures form due to gas instability, which could also explain some clumpy galaxies observed in the high-redshift Universe. These galaxies are referred to as clump clusters (chain galaxies). $[1,2]$ suggested that these clumpy stellar structures fall into the galactic centre by dynamical friction and merge into a single bulge at the galactic centre, a clump-origin bulge. Clump-origin bulges form through 'mergers of the clumps' in a galactic disc, neither the galactic merger nor the secular evolution. Therefore, properties of clump-origin bulges could be different from those of the conventional ones, classical bulges nor pseudobulges. I perform a similar numerical simulation to $[1,2]$ using an isolated halo model by a $N$-body/SPH code and study the naive natures of clump-origin bulges in details.

\section{RESULTS}

Our initial condition follows the spherical model that was used to study the formation of disc galaxies in an isolated environment. I assume an equilibrium system with the NFW profile with a virial mass

\footnotetext{
${ }^{a}$ e-mail: inoue@astr.tohoku.ac.jp
}

This is an Open Access article distributed under the terms of the Creative Commons Attribution-Noncommercial License 3.0, which permits unrestricted use, distribution, and reproduction in any noncommercial medium, provided the original work is properly cited. 

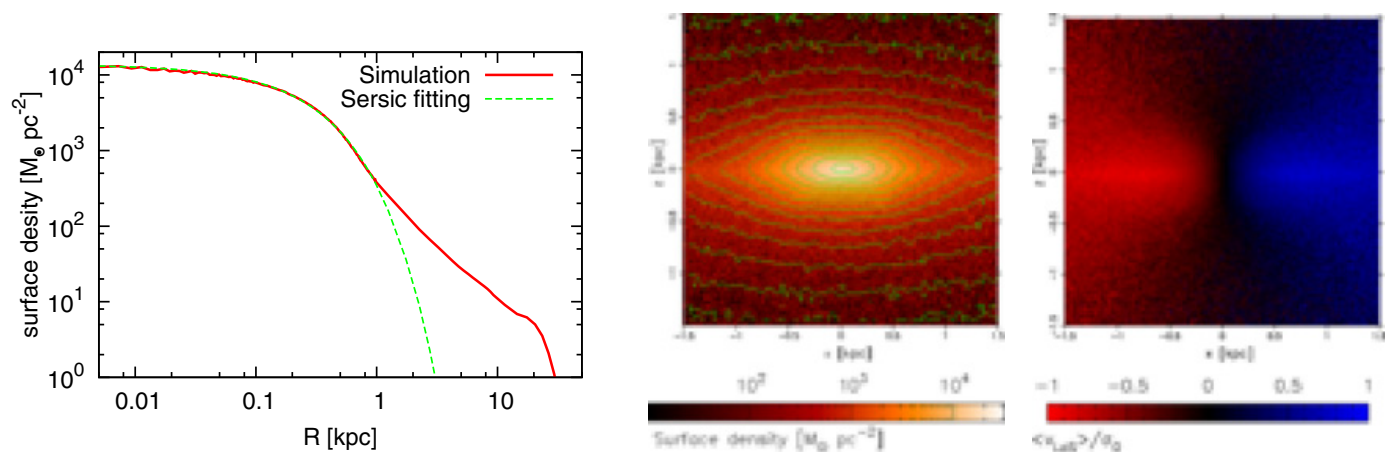

Figure 1. Stellar surface density profile and a fitting by the Sérsic profile (left). Stellar surface density maps in central $3 \times 3 \mathrm{kpc}$ region from the edge-on view (centre). Line-of-site velocity map of the stellar component (right).

$M_{\mathrm{vir}}=5.0 \times 10^{11} \mathrm{M}_{\odot}$. Baryon mass fraction of the system is set to 0.06 . The details of my simulation settings are given in $[4,5]$.

In Fig. 1, I plot the azimathally averaged surface density. The fitting by minimum $\chi^{2}$ is given by the Sersic index, $n=1.18$. This value of the index means the bulge has a nearly exponential density profile and indicates a pseudobulge-like density structure. As seen from the central panel, it clearly appears that this bulge is a boxy bulge. Ellipticity of the bulge measures 0.67 from the edgeon view. In the right panel, I illustrate a map of the mean line-of-sight velocity of stars from the edge-on view. The value is normalized by the velocity dispersion interior to the half mass radius of the bulge ( $450 \mathrm{pc}$ ), $\sigma_{0}=81.0 \mathrm{~km} \mathrm{~s}^{-1}$. This bulge shows significant rotation in the figure. The value of $V_{\max } / \sigma_{0} \simeq 0.9$ of this bulge means that the rotation (spin) is not negligible in kinematics but comparable to the dispersion, where $V_{\max }$ is the maximum mean LoS velocity in the bulge. These are indicating pseudobulge signatures [3].

From the simulation result, I found that the clump-origin bulge consists of stars with an over-solar metallicity [5]. Such a high metal abundance in the bulge is a natural outcome of the formation of clumps which form from a collapsing gas sphere in the highly gas-rich disc and the violent star formation in the clump mergers. Additionally, this bulge formation scenario, the clump cluster phase, is expected to happen only at the high-redshift. Therefore, the clump-origin bulge consists of old stars [5]. These old and metal-rich natures are similar to classical bulges rather than pseudobugles [3].

The MW bulge does not follow the properties of classical bulges nor pseudobulges. The MW bulge shows a nearly exponential profile, an oblate peanut shape (X-shape) and a significant rotation, which are similar to pseudobulges. At the same time, it has old stars, a high metallicity and weak star formation, which are similar to classical bulges [3]. These properties of the MW bulge are consistent with the clump-origin bulge obtained by our simulation. Such unclassifiable bulges like the MW bulge (old pseudobulge) are also observed in some other disc galaxies [6]. My simulation results suggest that clump-origin bulges could be a possible origin of these old pseudobulges.

We expect that the secular evolution would not be able to build a pseudobulge in early phase of the galaxy formation. It is because the 'secular' evolution takes a long time to form a pseudobulge. Therefore, we can expect that a galactic bulge observed in the high-redshift Universe would be either a classical bulge or a clump-origin bulge. The clump-origin bulge has a nearly exponential surface density profile, which is distinguishable from the $R^{1 / 4}$ profile of the classical bulges. Then, we would be able to identify the clump-origin bulges by identifying their exponential density profile. 
Assembling the Puzzle of the Milky Way

\section{References}

[1] Noguchi, M., Nat 392, (1998), 253

[2] Noguchi, M., ApJ 514, (1999), 77

[3] Kormendy, J. \& Kennicutt, Jr. R. C., ARA\&A 42, (2004), 603

[4] Inoue, S. \& Saitoh, T. R., astro-ph/1108.0906, (2011)

[5] Inoue, S. \& Saitoh, T. R., astro-ph/1109.2898, (2011)

[6] Bica, E. \& Alloin, D., A\&AS 70, (1987), 281 